
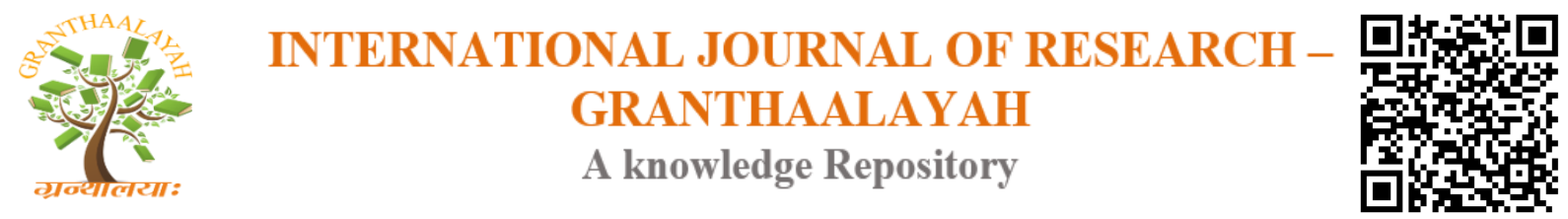

Science

\title{
KINETIC PROPERTY OF A PRESSURE VESSEL MADE FROM CFRP FABRICATED A FILAMENT WINDING METHOD
}

\author{
Kei-ichi OKUYAMA ${ }^{* 1}$, Shigeru HIBINO ${ }^{1}$, Aleksander LIDTKE ${ }^{1}$ \\ ${ }^{1}$ Applied Sciences and Integrated System Engineering, Kyushu Institute of Technology, 1-1 \\ Sensui-cho, Tobata Ward, Kitakyushu, Fukuoka Prefecture 804-0015, Japan
}

\begin{abstract}
Since the specific strength and the specific elasticity of carbon fiber reinforced plastics (CFRPs) are the greatest in practical materials, they are used abundantly in transport structures. This CFRP can also be used for a pressure vessel which stores liquid hydrazine, the required burst pressure is approximately 22MPa. Many researchers have been studying pressure vessels made from a CFRP fabricated by a filament winding (FW) method.

In order to acquire the fundamental mechanical properties of a CFRP container, the small cylinder made from CFRP fabricated by the spiral winding type FW method is designed and manufactured. The winding angle of a carbon fiber of this cylinder is \pm 45 degrees. The plastic deformation of this cylinder generates from the strain range of $0.7 \%$. So as to confirm by the analytical method that a small tank made from CFRP fabricated by the FW method can be utilized as a tank for liquid hydrazine, an analysis model is created. Since the pressure to which the strain of this CFRP tank reaches to $0.7 \%$ is approximately $35 \mathrm{MPa}$, this small tank can be used as a fuel tank for micro satellites.
\end{abstract}

Keywords: Micro Satellite; Filament Winding; CFRP; Fuel Tank.

Cite This Article: Kei-ichi OKUYAMA, Shigeru HIBINO, and Aleksander LIDTKE. (2018). "KINETIC PROPERTY OF A PRESSURE VESSEL MADE FROM CFRP FABRICATED A FILAMENT WINDING METHOD." International Journal of Research - Granthaalayah, 6(3), 140-148. https://doi.org/10.29121/granthaalayah.v6.i3.2018.1507.

\section{Introduction}

By integration of electronic components, such as a microcomputer, and adoption of materials with high specific strength and specific elasticity, such as carbon fiber reinforced plastics (CFRPs), the size of satellites continue to be miniaturized. Missions of satellites with a mass of $100 \mathrm{~kg}$ or less, which are called micro satellites, serve multiple uses, such as earth observation and deep space exploration. The orbital periods of stay of these satellites are becoming longer. The micro satellites must carry much thruster fuel for maintaining an orbital altitude and an attitude. The fuel currently used the most abundantly is liquid hydrazine, the pressures of the 
proof pressure and the burst pressure of a lightweight storage tank made from titanium alloy 6Al$4 \mathrm{~V}$ are $2.59 \mathrm{MPa}$ [375 $\mathrm{psi}$ ] and 3.10MPa [450 psi], respectively [1-2].

Since the specific strength and the specific elasticity of CFRPs are the greatest in practical materials, they are used abundantly in transport structures, such as automobiles, railroad vehicles, airplanes and spaceplanes. This CFRP can also be used for a pressure vessel which stores liquid hydrazine, the proof pressure and the burst pressure of lightweight storage tanks made from a CFRP fabricated by a filament winding (FW) method are 17.2MPa [2500 psi] and 21.6MPa [3125 psi], respectively [3]. Therefore, development of a small tank which can carry out a load-proof to the pressure of $30 \mathrm{MPa}$ is a trial which contributes to the miniaturization of a micro satellite.

Many researchers have been studying pressure vessels made from CFRP fabricated by a FW method. One of them purposes to establish production methods of liquid or gas containers which adopted CFRP for both a liner and an outer shell.

In this research, the main purpose is to acquire the fundamental mechanical properties of a container made from CFRP fabricated by a FW method, and this container can be used it under high pressure up to $30 \mathrm{MPa}$ and is also evaluated by an analytical method.

\section{Mechanical Property of a Pressure Vessel Made From Cfrp Fabricated By a Fw Method}

Filament-winding-molding methods, i.e. FW methods have a parallel winding method, a level winding method and a spiral winding method, those outlines are shown in Fig. 1. In this research, we adopted the spiral winding method. As for this spiral winding method, a uniformly distributed load acts on the $x$-axis to which angle $\theta$ inclined to the fibers. This is shown in Fig. 2 in simple.

(a)



(b)



(c)

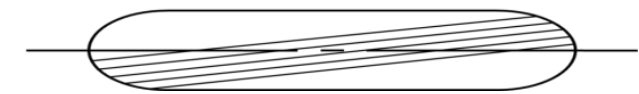

Figure 1: Filament-winding-molding method, (a) parallel winding method, (b) spiral winding method and (c) level winding method

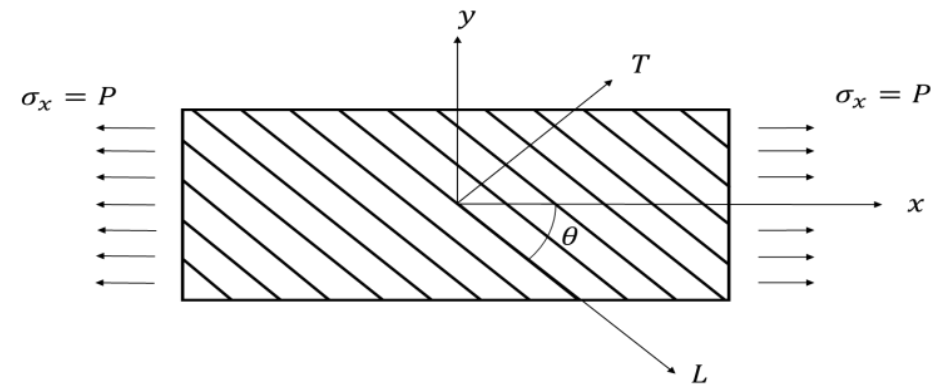

Figure 2: Orthotropic anisotropy board on which load acted in x-direction 
When Hooke's law about the plane stress of a one-way reinforced material is used, the relation between stress and strain about the elastic axis of symmetry is expressed by Eq. 1,

$\left[\begin{array}{c}\sigma_{x} \\ \sigma_{y} \\ \tau_{x y}\end{array}\right]=\left[\begin{array}{lll}C_{11} & C_{12} & C_{13} \\ C_{21} & C_{22} & C_{23} \\ C_{31} & C_{32} & C_{33}\end{array}\right]\left[\begin{array}{c}\varepsilon_{x} \\ \varepsilon_{y} \\ \varepsilon_{z}\end{array}\right]$,

Where $\sigma$ and $\varepsilon$ are stress and strain, the coefficient $C s$ are expressed by from Eq. 2 to Eq. 7 .

$$
\begin{aligned}
& C_{11}=E_{L}^{\prime} \cos ^{4} \theta+E_{T}^{\prime} \sin ^{4} \theta+2 E_{L}^{\prime} \nu_{T L} \cos ^{2} \theta \sin ^{2} \theta+4 G_{L T} \cos ^{2} \theta \sin ^{2} \theta^{2} \\
& C_{22}=E_{L}^{\prime} \sin ^{4} \theta+E_{T}^{\prime} \cos ^{4} \theta+2 E_{L}^{\prime} v_{T L} \cos ^{2} \theta \sin ^{2} \theta+4 G_{L T} \cos ^{2} \theta \sin ^{2} \theta \\
& C_{33}=E_{L}^{\prime}\left(1-v_{T L}\right) \cos ^{2} \theta \sin ^{2} \theta+E_{T}^{\prime}\left(1-v_{L T}\right) \cos ^{2} \theta \sin ^{2} \theta+\left(\cos ^{2} \theta-\sin ^{2} \theta\right)^{2} G_{L T}, \\
& C_{12}=C_{21}=E_{T}^{\prime}\left(\cos ^{2} \theta-v_{T L} \sin ^{2} \theta\right) \cos ^{2} \theta+E_{L}^{\prime}\left(\cos ^{2} \theta+v_{L T} \sin ^{2} \theta\right) \sin ^{2} \theta \\
& -4 G_{L T} \cos ^{2} \theta \sin ^{2} \theta,
\end{aligned}
$$

$C_{23}=C_{32}=-E_{L}^{\prime}\left(1-v_{T L}\right) \cos \theta \sin ^{3} \theta+E_{T}^{\prime}\left(1-v_{L T}\right) \cos ^{3} \theta \sin \theta+$

$2 G_{L T}\left(\cos ^{2} \theta-\sin ^{2} \theta\right)^{2} \cos \theta \sin \theta$,

Where

$E_{L}^{\prime}=\frac{E_{L}}{1-v_{L T} v_{T L}}, \quad E_{T}^{\prime}=\frac{E_{T}}{1-v_{L T} v_{T L}}$,

Where $E, G$ and $v$ are the elastic modulus, the shear modulus and Poisson's ratio.

Eq. 1 is rewritten about strain,

$$
\left[\begin{array}{c}
\varepsilon_{x} \\
\varepsilon_{y} \\
\gamma_{x y}
\end{array}\right]=\left[\begin{array}{lll}
S_{11} & S_{12} & S_{13} \\
S_{21} & S_{22} & S_{23} \\
S_{31} & S_{32} & S_{33}
\end{array}\right]\left[\begin{array}{c}
\sigma_{x} \\
\sigma_{y} \\
\sigma_{z}
\end{array}\right],
$$

Where, $S_{i j}$ is called a Moduli of compliance, which is expressed by Eq. 10 to Eq. 15,

$$
S_{11}=\frac{1}{E_{L}} \cos ^{4} \theta+\frac{1}{E_{T}} \sin ^{4} \theta+2\left(\frac{1}{G_{L T}}-\frac{2 v_{L T}}{E_{L}}\right) \cos ^{2} \theta \sin ^{2} \theta
$$




$$
\begin{aligned}
& S_{22}=\frac{1}{E_{L}} \sin ^{4} \theta+\frac{1}{E_{T}} \cos ^{4} \theta+2\left(\frac{1}{G_{L T}}-\frac{2 v_{L T}}{E_{L}}\right) \cos ^{2} \theta \sin ^{2} \theta \\
& S_{33}=\frac{1}{G_{L T}}+4\left(\frac{1}{E_{L}}+\frac{1}{E_{T}}+\frac{2 v_{L T}}{E_{L}}-\frac{1}{G_{L T}}\right) \cos ^{2} \theta \sin ^{2} \theta \\
& S_{12}=S_{21}=-\frac{v_{L T}}{E_{L}}+\left(\frac{1}{E_{L}}+\frac{1}{E_{T}}+\frac{2 v_{L T}}{E_{L}}-\frac{1}{G_{L T}}\right) \cos ^{2} \theta \sin ^{2} \theta \\
& S_{13}=S_{31}=-2\left(\frac{1+v_{L T}}{E_{L}}\right) \cos ^{3} \theta \sin \theta+2\left(\frac{1+v_{L T}}{E_{L}}\right) \cos \theta \sin ^{3} \theta+\frac{\left(\cos ^{2} \theta-\sin ^{2} \theta\right)^{2} \cos \theta \sin \theta}{G_{L T}} \\
& S_{23}=S_{32}=-2\left(\frac{1+v_{L T}}{E_{L}}\right) \cos \theta \sin ^{3} \theta+2\left(\frac{1+v_{L T}}{E_{L}}\right) \cos \theta \sin ^{3} \theta-\frac{\left(\cos ^{2} \theta-\sin ^{2} \theta\right)^{2} \cos \theta \sin \theta}{G_{L T}}
\end{aligned}
$$

In Fig. 2, the $x$-axis is the elastic axis of symmetry, and the uniformly distributed load $P$ is acting on this $x$-axis. The relation between the stress and the strain is expressed by Eq. 1,

$\sigma_{x}=P, \sigma_{y}=0, \tau_{x y}=0$

Using Eq. 9, Eq. 16 can be rewritten as Eq. 17 to Eq. 19,

$$
\begin{gathered}
\varepsilon_{x}=S_{11} \sigma_{x}=S_{11} P, \\
\varepsilon_{x}=S_{12} \sigma_{x}=S_{12} P \\
\tau_{x y}=S_{13} \sigma_{x}=S_{13} P .
\end{gathered}
$$

Therefore, the elastic modulus on $x$-axis and $y$-axis are expressed as:

$$
\begin{gathered}
1 / E_{x}=S_{11}=\frac{\cos ^{4} \theta}{E_{L}}+\frac{\sin ^{4} \theta}{E_{T}}+\left(\frac{1}{G_{L T}}-\frac{2 v_{L T}}{E_{L}}\right) \cos ^{2} \theta \sin ^{2} \theta \\
1 / E_{y}=S_{12}=-\frac{v_{L T}}{E_{L}}+\left(\frac{1}{E_{L}}+\frac{1}{E_{T}}+\frac{2 v_{L T}}{E_{L}}-\frac{1}{G_{L T}}\right) \cos ^{2} \theta \sin ^{2} \theta .
\end{gathered}
$$

\section{Validation Tests}

In order to confirm the validity of the evaluation method shown in the 2 nd chapter, the tensile test of a cylindrical container was carried out. The model used this test is a cylinder made from CFRP fabricated by the spiral winding type FW method with $64 \mathrm{~mm}$ on the outside diameters, $100 \mathrm{~mm}$ in the height, and the thickness of $2 \mathrm{~mm}$. This model was fabricated by Chugoku Kogyo Co., Ltd.

The winding angle of carbon fiber on the cylinder used for this research is \pm 45 degrees. The CFRP fiber is UT500 of Toho Tenax Co., Ltd. and the epoxy resin is XNR6805 of Nagase Chemtex Co., Ltd. which are used in this study. The elastic modulus of the carbon fiber UT500 
and the epoxy resin XNR6805 are 240GPa and 3.4GPa [4]. The fiber content $V_{f}$ and the resin content $V_{m}$ of the CFRP are approximately 0.5 , respectively.

The elastic modulus of the direction of the longitudinal direction and the lateral direction of a laminate sheet are calculable by Eq. 22 to Eq. 26 [5].

$G_{f}=E_{f} / 2\left(1-v_{f}\right)$

$G_{m}=E_{m} / 2\left(1-v_{m}\right)$,

$G_{L T, \text { longitudinal }}=G_{f} G_{m} /\left[G_{m} V_{f}+G_{f}\left(1-V_{f}\right)\right]$,

$G_{\text {LT,lateral }}=G_{f} V_{f}-G_{m}\left(1-V_{f}\right)$,

$G_{L T}=(1-c) G_{L T, \text { longitudinal }_{f}}-c G_{L T, \text { lateral }}$

where subscripts $f$ and $m$ are a fiber and a resin. $c$ is called the contiguous coefficient which are expressed as [5]:

$c=0.2\left(V_{f}-V_{m}\right)+0.175$.

The appearance of the cylinder type specimen is shown in Fig. 3. Tensile test specimens were cut down from these cylinder models. The specimen for tensile tests is shown in Fig. 4. The small desk type testing equipment, EZTest of the maximum load 5000N of Shimadzu Corporation, was used for the tests.

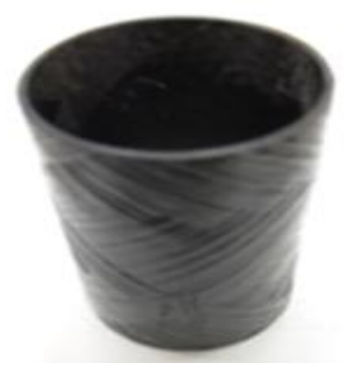

Figure 3: CFRP cylinder which was manufactured by the filament-winding-method (Fiber winding angles are $\pm 45^{\circ}$ )
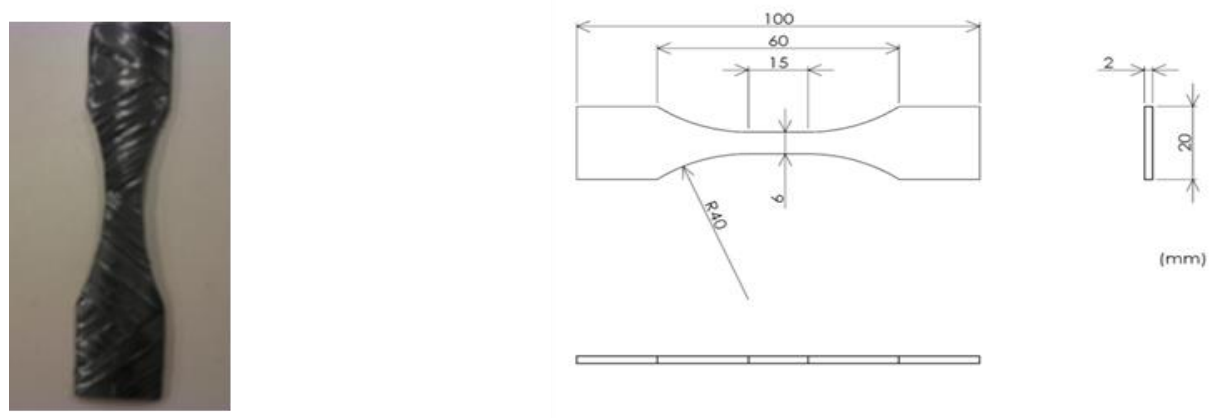

Figure 4: Specimen for a tension test which was cut down from the CFRP circular 


\section{Results and Discussions}

The test using the specimens of Fig. 4 are carried out 4 times, the tensile elastic modulus and the tensile strengths are measured. The test results are shown in Fig. 5. Moreover, the enlargement of a fracture side is shown in Fig. 6.

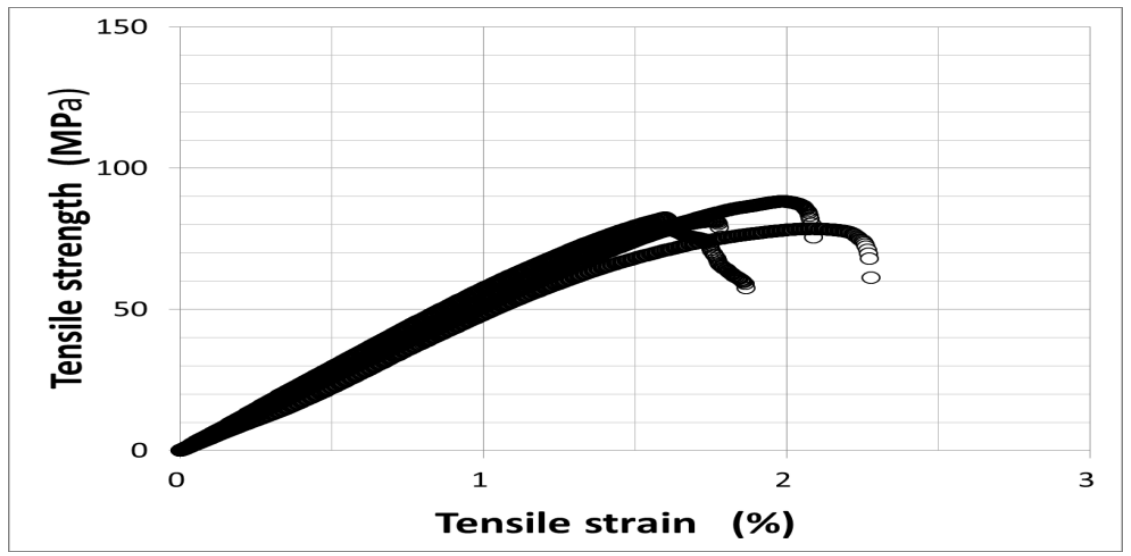

Figure 5: Relation between the stress and the strain to have gotten from the tension test (fiber rolling angle is $\pm 45^{\circ}$ )

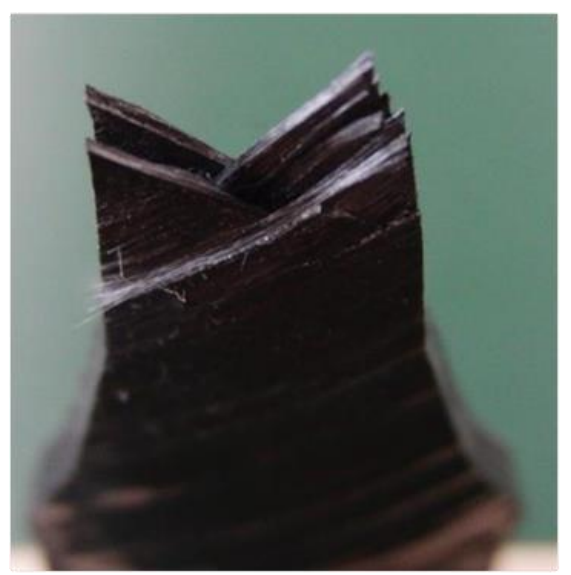

Figure 6: fracture surface of a specimen after tension test (fiber rolling angle is $\pm 45^{\circ}$ )

1) The tensile strength and the tensile elastic modulus of the CFRP cylinder fabricated by the FW with the fiber winding angle of \pm 45 degrees are $83 \mathrm{MPa}$ and 5.1GPa, respectively.

2) Since the mechanical properties of the cylinder shown in Fig. 3 are considered to be directions, such as a quasi-isotropic, $E_{x}$ and $E_{y}$ of the cylinder winded at \pm 45 degrees are $5.1 M P a$, respectively.

3) The strains in the elastic limit region are located between $0.7 \%$ and $1.3 \%$.

4) From Fig. 7, it can be understood that the destruction originated in the L-direction.

Because $V_{f}$ and $V_{m}, E_{f}$ and $E_{m}, E_{L}$ and $E_{T}$ of the CFRP cylinder used for this research are 0.5 and $0.5,240 G P a$ and $3.4 G P a, 122 G P a$ and $29.7 G P a$, respectively [4-5]. $c$ is calculated to 0.175 . However, Tsai et al. found out that an analysis value and an experimental value corresponded 
when c is set to approximately 0.2 based on the Hashin et al.'s analysis results [6-7]. As for the value of $c, 0.2$ is adopted in this research. In this research, Poisson's ratio of $L-T$ plane $\nu_{L T}$ was set to 0.30 . Therefore, $G_{L T}$ can be calculated by having substituted these values for Eq. 20 and Eq. 21 that is $1.35 G P a$. These values are conclusions in Table 1.

Table 1: Mechanical properties of CFRP cylinder fabricated by FW method

\begin{tabular}{|c|c|c|c|c|c|c|c|}
\hline \multirow{2}{*}{ Volume angle of fiber } & \multicolumn{3}{|l|}{ Values about fiber and resin } & \multicolumn{2}{|c|}{ Values about principal axis } & \multicolumn{3}{|c|}{ Values about fibrous directions } \\
\cline { 2 - 8 } & \multicolumn{2}{|c|}{ Elastic modulus } & \multicolumn{2}{|c|}{ Elastic modulus } & \multicolumn{2}{|c|}{ Elastic modulus } & $\begin{array}{c}\text { Shear } \\
\text { modulus }\end{array}$ \\
\hline \multirow{2}{*}{ degree ] } & $E_{f}$ & $E_{m}$ & $E_{x}$ & $E_{y}$ & $E_{L}$ & $E_{T}$ & $G_{L T}$ \\
\cline { 2 - 8 } & {$[G P a]$} & {$[G P a]$} & {$[G P a]$} & {$[G P a]$} & {$[G P a]$} & {$[G P a]$} & {$[G P a]$} \\
\hline 45 & 240 & 3.40 & 5.10 & 5.10 & 122 & 29.7 & 1.35 \\
\hline
\end{tabular}

Evaluations of a CFRP container fabricated by the FW method which can be used under high pressure up to $30 \mathrm{MPa}$ is also one of the purposes of this research. In order to carry this out, we examined by an analytical method, a tank made from CFRP fabricated by the FW method which can be applied to a high-pressure container using this study's results. The finite element analysis software ABAQUS (Version 6.8-1) is used for the analysis, a large deformation contact analysis method is adopted. The full length, the outside radius, the inner radius, and liner thickness of this tank are $225 \mathrm{~mm}, 70 \mathrm{~mm}, 50 \mathrm{~mm}$, and $3.5 \mathrm{~mm}$, respectively. The outer shell is made from CFRP fabricated by the FW method, the liner CFRP, is a composite material fabricated by laminating prepregs. The CFRP employed in this study is comprised of an epoxy resin and polyacrylonitrile (PAN) fibers. PAN yarn with a diameter of $7 \mu \mathrm{m}$ is twisted into bundles of 3000 filaments, and these bundles are used to make the PAN fiber. The elastic modulus and Poisson's ratio of this liner CFRP are 138GPa and 0.33. The cap is SUS316 alloy, the elastic modulus and Poisson's ratio are $193 \mathrm{GPa}$ and 0.29 . The analysis model is shown in Fig. 7. This analysis was performed on the conditions from which the external pressure is constant $0.1 \mathrm{MPa}$, the inside pressure changes by $5 \mathrm{MPa}$ unit from $0.1 \mathrm{MPa}$ to $70 \mathrm{MPa}$. Analysis results are shown in Fig. 8.

J. Park et al. did practical research about CFRP motor case fabricated by a FW method for a rocket [8]. J. Park et al. found out that the maximum cracking of this tank made from CFRP occurs near a metal cap.

In this analytical research, the metal cap circumference is investigated carefully.
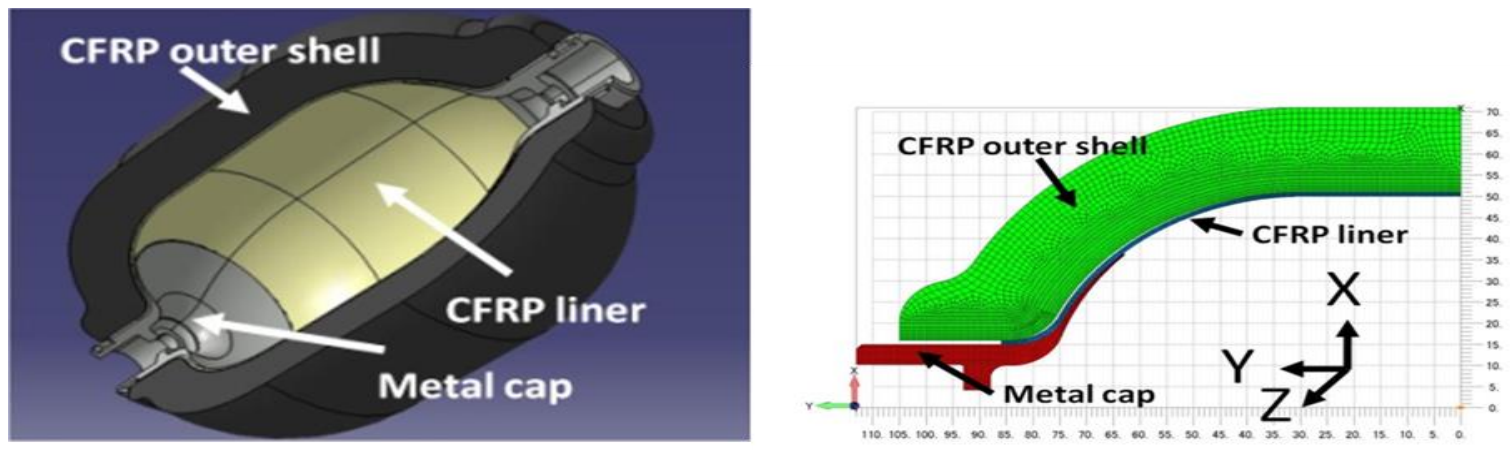

Figure 7: Finite-element-method analysis model of CFRP container fabricated by FW method 


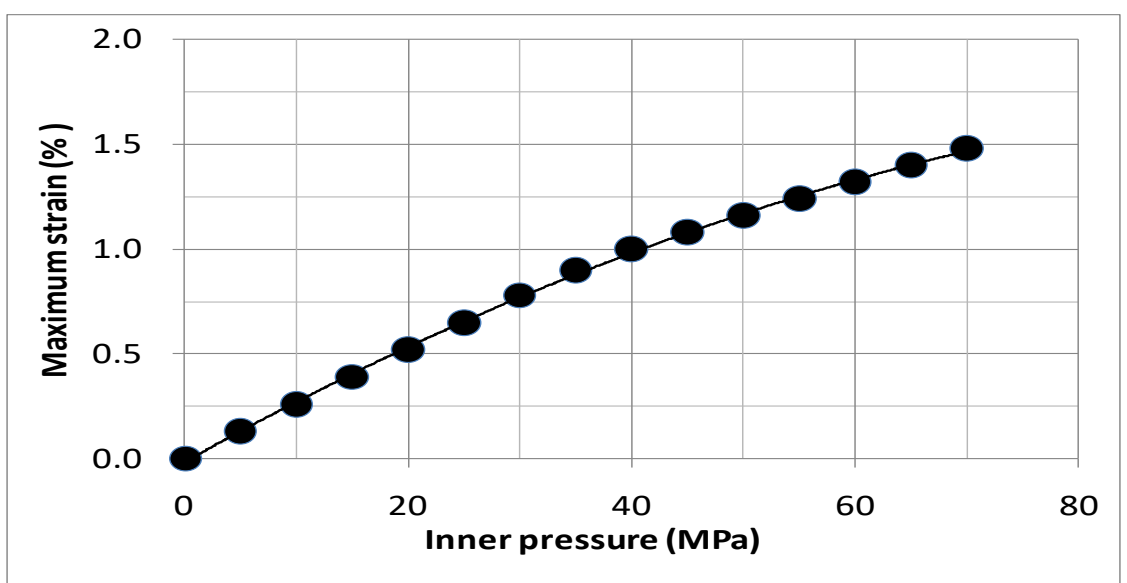

Figure 8: Relation between the maximum strain analysis values and the internal pressure around the CFRP tank cap part

Fig. 5 shows that the plastic deformation generates from the strain range from $0.7 \%$. Fig. 8 shows that pressure is approximately $35 \mathrm{MPa}$ while reaching the plastic deformation.

It can be understood with Fig. 8 that the pressure to which the maximum for the plastic deformation of this tank exceeds $0.7 \%$ is approximately $35 \mathrm{MPa}$. This CFRP tank fabricated by this FW method can be used up to approximately $35 \mathrm{MPa}$.

In order to develop a small liquid hydrazine tank for transport vehicles, the mechanical properties of the CFRP cylinder fabricated by the FW were acquired. A small CFRP tank can be used as a high-pressure tank using acquired physical properties were evaluated by an analytical method, these results are shown below.

Since the specific strength and the specific elasticity of CFRPs are the greatest in practical materials, they are used abundantly in transport structures. This CFRP can also be used for a pressure vessel which stores liquid hydrazine, the proof pressure and the burst pressure of lightweight storage tanks made from CFRP fabricated by a FW method are approximately $17 M P a$ and $22 M P a$, respectively.

In order to acquire the fundamental mechanical properties of a CFRP container. A cylinder made from a CFRP fabricated by the spiral winding type FW method with $64 \mathrm{~mm}$ of the outside diameters, $100 \mathrm{~mm}$ in the height, and the thickness of $2 \mathrm{~mm}$ is designed and manufactured. The winding angle of a carbon fiber of this cylinder is \pm 45 degrees. The fiber content $V_{f}$ and the resin content $V_{m}$ of the CFRP are approximately 0.5 , respectively. The elastic modulus $E_{x}$ and Poisson's ratios $\nu_{x}$ in $x$-y plane are used 5.1GPa and 0.32 in this research. Since the winding angle of the carbon fiber is 45 degrees, it is thought that $E_{y}$ and $v_{y}$ are equivalent to $E_{x}$ and $v_{x}$. The plastic deformation of this CFRP cylinder generates from the strain range from $0.7 \%$.

To confirm by analytical method that a small tank made from CFRP fabricated by the FW method can be utilized as a tank for liquid hydrazine, an analysis model is created. The full length, the outside radius, the inner radius, and liner thickness of this tank are $225 \mathrm{~mm}, 70 \mathrm{~mm}$, $50 \mathrm{~mm}$, and $3.5 \mathrm{~mm}$, respectively. Since the pressure to which the strain of this CFRP tank reaches $0.7 \%$ is approximately $35 \mathrm{MPa}$, this small tank can be used as a fuel tank for micro satellites. 


\section{References}

[1] Paul S. Griffin, Ian A. Ballinger, Donald E. Jaekle Jr. and Arthur C. Jackson III, DESIGN AND MANUFACTURE OF A LIGHTWEIGHT FUEL TANK ASSEMBLY, AIAA-2003-4606, 39th AIAA PROPULSION CONFERENCE, 21 July 2003, Alabama (USA).

[2] Walter Tam, Mike Hersh, and Ian Ballinger, HYBRID PROPELLANT TANKS FOR SPACECRAFT AND LAUNCH VEHICLES, AIAA-2003-4607, 39th AIAA PROPULSION CONFERENCE, 21 July 2003, Alabama (USA).

[3] N Walter H. Tam, Michael J. Debreceni, Michael S. Hersh and Charles D. Nye, LOW COST DERIVATIVE TANKS FOR SPACECRAFT AND LAUNCH VEHICLES, 35th AIAA/ASME/SAE/ASEE JOINT PROPULSION CONFERENCE, 20-24 July 1999, California (USA).

[4] M S. Okamoto1, M. Yamamoto, K. Hosokawa, M. Nakano and K. Hanaoka, MECHANICAL PROPERTIES OF CFRP PRESSURE VESSELS AND IDENTIFICATION OF EQUIVALENT ELASTIC MODULI OF CFRPS, WIT Transactions on The Built Environment, Vol 97 (2008), pp. 471-480.

[5] M. Uemura, K. Yamawaki, S. Abe and H. Iyama, ABOUT THE RIGIDITY OF A FILAMENT WINDING MATERIAL, BULLETIN OF THE INSTITUTE OF SPACE AND AERONAUTICAL SCIENCE, University of Tokyo, Vol.4, No.3(B)(1968), pp. 448-463 (in Japanese).

[6] Stephen W Tsai, STRUCTURAL BEHAVIOR OF COMPOSITE MATERIALS, National Aeronautics and Space Administration contractor report NASA CR-71 (1964), Washington (USA).

[7] Zvi Hashin and B. Walter Rosen, THE ELASTIC MODULI OF FIBER-REINFORCED MATERIALS, Journal of Applied Mechanics, Vol. 31, Issue 2 (1964), pp. 223-232.

[8] Jae-Sung Park, Cheol-Ung Kim, Hyun-Kyu Kang, Chang-Sun Hong and Chun-Gon Kim, STRUCTURAL ANALYSIS AND STRAIN MONITORING OF THE FILAMENT WOUND MOTOR CASE, Journal of Composite Materials, Vol. 36, No. 20 (2002), pp. 2373-2388.

\footnotetext{
*Corresponding author.

E-mail address: okuyama@ ise.kyutech.ac.jp
} 